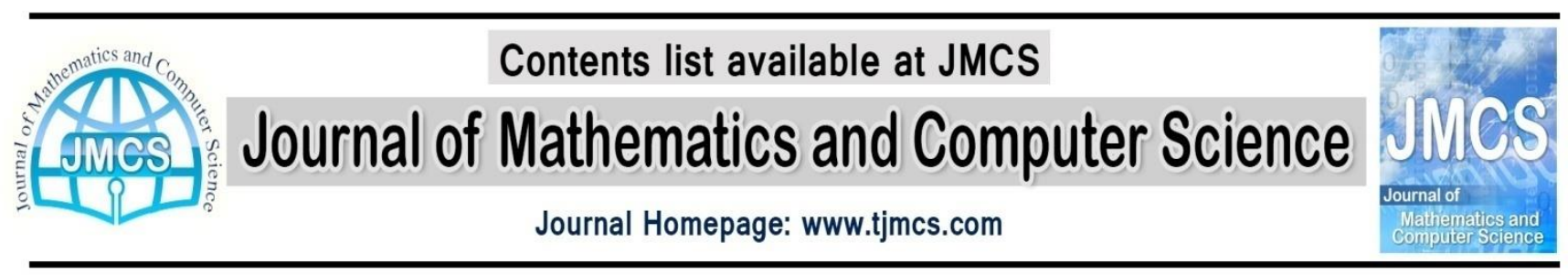

\title{
An Asymptotic Stability Criteria of Delay Differential Equations on Time Scals
}

\author{
Jamal Saffar Ardabili, ${ }^{1, *}$, Zahra Poursepahi Samian",+ \\ ${ }^{1}$ Department of Mathematics, Payame Noor University, P. O. Box. 19395-3697, Tehran, Iran. \\ "saffar@pnu.ac.ir \\ +z.poursepahi@yahoo.com
}

\section{Article history: \\ Received November 2014 \\ Accepted February 2015 \\ Available online February 2015}

\section{Abstract}

Let $T$ be an arbitrary time scale that is unbounded above. In this paper, we will present some stability criteria for first order delay differential equations

$$
x^{\Delta}(t)=a(t) x(t)+b(t) x(\tau(t))
$$

using their asymptotic behavior.

\section{AMS Mathematics Subject Classification: 34K20, 39A30}

Keywords: Delay differential equations, Time scale, Asymptotic behavior, Stability.

\section{Introduction}

Time delays occur in many social and natural phenomena, often in a form of time gap between an action/decision and the corresponding effects. Time delays also exist in different systems such as mechanical systems, chemical systems, etc.

A major class in dynamical systems is delay differential equations. These equations particularly arise in control problems. In a control problem, the state of the system is continuously monitored by a controller and the system is adjusted after each monitoring process, based on the observations. Here, as a result of the gap between monitoring and adjustment, a considerable time delay can occur.

A good example is the Insulin-Glucose system. The most important factors in this system are the amount of Insulin, Glucose and their alteration rate. The system is represented with a delay differential equation with two delays. Writing the mass conservation law for both Insulin and Glucose, we have: 


$$
\left\{\begin{array}{l}
G^{\prime}(t)=G_{i n}-f_{2}(G(t))-f_{3}(G(t)) f_{4}(G(t))+f_{5}(G(t)) \\
I^{\prime}(t)=f_{1}\left(G\left(t-\tau_{1}\right)\right)-d_{i} I(t)
\end{array}\right.
$$

with the initial conditions

$$
\begin{cases}I(0)=I_{0}>0, & \\ G(0)=G_{0}>0, \quad G(t) \equiv G_{0} & ; t \in\left[-\tau_{1}, 0\right], \tau_{1}>0 \\ I(t) \equiv I_{0} & ; t \in\left[-\tau_{2}, 0\right], \tau_{2}>0 .\end{cases}
$$

In this relation we present the production of Hepatic Glucose with $f_{5}(I)$, that is related to blood Insulin, the rate of glucose consumption with $f_{2}(G)$ and the absorption with respect to Insulin with $f_{3}(G) f_{4}(I)$.

\section{Some Preliminaries}

\subsection{Time Scale}

Despite the great importance of studying time delays, the theory of time delay equations had not been developed until 1990. The study of time delay systems which was very difficult in the infinitedimensional space, became easy after developments in nonlinear dynamics.

The theory of time scales was introduced by Stefan Hilger in 1988. His main purpose was to unify continuous and discrete analysis (see [1]). This theory is able to unify the theories of differential equations and difference equations. Besides, it can extend those classical cases to cases in between.

Definition 2.1 ( time scale). A time scale $T$ is an arbitrary nonempty closed subset of $R$.

The cases when this time scale is equal to the real numbers or to the integer numbers represent the classical theories of differential and of difference equations. Many other interesting time scales exist, and they give rise to plenty of applications.

A book on the subject of time scale by Bohner and Peterson [2,9] summarizes and organizes much of time scale calculus. For the notions used below we refer to [2-10]. In the next section we recall some of the main tools used in the subsequent sections of this paper.

Definition 2.2 let $T$ be a time scale. For $t \in T$ we define the forward jump operator $\sigma: T \rightarrow T$ by

$$
\sigma(t):=\inf \{s \in T: s>t\}
$$

Definition 2.3 let $T$ be a time scale. For $t \in T$ we define the backward jump operator $\rho: T \rightarrow T$ by

$$
\rho(t):=\sup \{s \in T: s<t\} .
$$

Definition 2.4 If $\sigma(t)>t$, we say that $t$ is right-scattered, while if $\rho(t)<t$ we say that $t$ is leftscattered. 
Definition 2.5 If $t<\sup (T)$ and $\sigma(t)=t$, we say that $t$ is right-dense, and if $t>\operatorname{int}(T)$ and $\rho(t)=t$ we say that $t$ is left-dense.

Definition 2.6 The graininess function $\mu: T \rightarrow R$ is defined by

$$
\mu(t):=\sigma(t)-t
$$

Definition 2.7 If $T$ has a left-scattered maximum $m$, then $T^{\kappa}=T-m$. Otherwise, $T^{\kappa}=T$. In summary,

$$
T^{\kappa}=\left\{\begin{array}{cl}
T-[(\rho(\sup T), \sup T] & , \sup T<\infty \\
T & , \sup T=\infty .
\end{array}\right.
$$

Definition 2.8 If $f: T \rightarrow R$ is a function, when we define the function $f^{\sigma}: T \rightarrow R$ by

$$
f^{\sigma}(t)=f(\sigma(t))=f o \sigma(t)
$$

Example 2.9 In the following table, we get some examples:

\begin{tabular}{|c|c|c|c|}
\hline time scale & $\sigma$ & $\rho$ & $\mu$ \\
\hline$T=R$ & $\sigma(t)=t$ & $\rho(t)=t$ & $\mu(t)=0$ \\
\hline$T=Z$ & $\sigma(t)=t+1$ & $\rho(t)=t-1$ & $\mu(t)=1$ \\
\hline$T=[a, b]$ & $\sigma(t)=t$ & $\rho(t)=t$ & $\mu(t)=0$ \\
\hline$T=h Z$ & $\sigma(t)=t+h$ & $\rho(t)=t-h$ & $\mu(t)=h$ \\
\hline
\end{tabular}

Definition 2.10 Assume $f: T \rightarrow R$ is a function and let $t \in T^{K}$. Then, we define $f^{\Delta}(t)$ to be a number (provided it exists) with the property that given any $\varepsilon>0$, there exist a neighborhood $U$ of $t$, such that

$$
\forall t \in T \quad \forall s \in U \quad\left|[f(\sigma(t))-f(s)]-f^{\Delta}(t)[\sigma(t)-s]\right| \leq \varepsilon|\sigma(t)-s|
$$

We call $f^{\Delta}(t)$ the delta (or Hilger) derivative of $f$ at $t$.

Theorem 2.11 (see [2]). Assume $f: T \rightarrow R$ is a function and let $t \in T^{\kappa}$. Then we have the following:

1. If $f$ is differentiable at $t$, then $f$ is continuous at $t$.

2. If $f$ is continuous at $t$ and $t$ is right-scattered, then $f$ is differentiable at $t$ with

$$
f^{\Delta}(t)=\frac{f(\sigma(t))-f(t)}{\mu(t)} .
$$


3. If $f$ is right-dense, then $f$ is differentiable at $t$ iff the

$$
\lim _{s \rightarrow t} \frac{f(t)-f(s)}{t-s}
$$

exists as a finite number. In this case

$$
f^{\Delta}(t)=\lim _{s \rightarrow t} \frac{f(t)-f(s)}{t-s} .
$$

4. If $f$ is differentiable at $t$, then

$$
f(\sigma(t))=f(t)+\mu(t) f^{\Delta}(t)
$$

Definition 2.12 A function $f: T \rightarrow R$ is called regulated provided its right-sided limits exist (finite) at all right-dense points in $T$ and its left-sided limits exist (finite) at all left-dense points in $T$.

Definition 2.13 A function $f: T \rightarrow R$ is called $r d$-continuous provided it is continuous at right-dense points in $T$ and its left-sided limits exist (finite) at all left-dense points in $T$. The set of rd-continuous functions $f: T \rightarrow R$ will denoted by $C_{r d}(T)$.

Definition 2.14 A function $f: T \rightarrow R$ is called regressive

$$
1+\mu(t) f(t) \neq 0
$$

The set of all function $f: T \rightarrow R$ that are regressive will be denoted by $R$. Also the set of all positively regressive function, that is

$$
\{f \in R: 1+\mu(t) f(t) \geq 0, \text { forallt } \in T\}
$$

will be denoted by $R^{+}$.

Definition 2.15 If $p \in R$, then we define the exponential function by

$$
e_{p}\left(t, t_{0}\right):=y(t)=\exp \left\{\int_{t_{0}}^{t} \zeta_{\mu(s)}(p(s)) \Delta s\right\} y\left(t_{0}\right)
$$

for all $s, t \in T$, where $\zeta_{h}(z)$ is the cylinder transformation, which is given by

$$
\zeta_{h}(z)= \begin{cases}\frac{\log (1+h z)}{h} & , h \neq 0 \\ z & , h=0\end{cases}
$$

Theorem 2.16 ( Mean Value Theorem(see [2])). Let $f$ be continuous on $[c, d]$ and delta-differential on $[c, d)$, where $c, d \in T, c<d$. Then there exist $a, b \in[c, d)$ such that 


$$
f^{\Delta}(a) \leq \frac{f(d)-f(c)}{d-c} \leq f^{\Delta}(b)
$$

Theorem 2.17 (Chain Rule(see [2])). Assume that $f: T \rightarrow R$ is increasing, $T:=f(T)$ is a time scale and let $g:=T \rightarrow R$. If $f^{\Delta}$ and $g^{\tilde{\Delta}}(f(t))$ exist for all $t \in T$, where " $\tilde{\Delta} "$ is the delta-derivative on $T$, then

$$
\forall t \in T, \quad(g \circ f)^{\Delta}(t)=g^{\tilde{\Delta}}(f(t)) f^{\Delta}(t)
$$

Theorem 2.18 (Substitution(see [2])). Let $f \in C_{r d}^{1}(T)$ be an increasing function, $T:=f(T)$ be a time scale and let $g \in C_{r d}(T)$. Then

$$
\forall c, d \in T, \quad \int_{c}^{d} g(t) f^{\Delta}(t) \Delta t=\int_{f(c)}^{f(d)}\left(g \circ f^{-1}\right)(s) \tilde{\Delta}(s)
$$

Theorem 2.19 (see [2]). Let $y, f \in C_{r d}$ and $p \in R^{+}$. Then

$$
\forall t \in T \quad y^{\Delta}(t) \leq p(t) y(t)+f(t)
$$

implies

$$
\forall t \in T \quad y(t) \leq y\left(t_{0}\right) e_{p}\left(t, t_{0}\right)+\int_{t_{0}}^{t} e_{p}(t, \sigma(t)) f(\tau) \Delta \tau
$$

\subsection{Stability}

In [3] M. Adivar and Y. Raffoul represent some stability criteria for first order delay differential equations

$$
x^{\Delta}(t)=b(t) x(t)-a(t) x(\tau(t)) \tau^{\Delta}(t)
$$

with the use of Lyapunov theorem and fixed point theory. Also, Kipnis and Levitskaya in [4], present some stability criteria for first order differential and difference equation.

First, we get some definitions for the stability of solutions of an arbitrary differential equation.

Definition 2.20 Let $x(t)$ be a solution of the system

$$
x^{\Delta}(t)=b(t) x(t)-a(t) x(\tau(t)) \tau^{\Delta}(t)
$$

we say that $x(t)$ is asymptotically stable if

$$
\lim _{t \rightarrow \infty} x(t)=0
$$




\subsection{The Asymptotic Behavior}

Throughout the paper, we suppose that the time scale under consideration is not bounded above, i.e. $\sup T=\infty$.

First, we are looking for some relations to study stability of solutions of first order delay differential equations. Next, we introduce some auxiliary functional relations which turn out to be of great importance in the asymptotic investigation of

$$
x^{\Delta}(t)=a(t) x(t)+b(t) x(\tau(t))
$$

We consider the equation

$$
\forall t \in T, \quad \psi(\tau(t))=\psi(t)-1,
$$

and the inequality

$$
\forall t \in T, \quad|b(t)| \phi(\tau(t)) \unlhd a(t) \mid \phi(t)
$$

The simple result ensuring the existence of a solution of (6) have certain delta-differential properties.

Proposition 2.21 (see [5]). Assume that the function $\tau \in C_{r d}^{1}(T)$ satisfies $\tau(t)<t$ for all $t \in T, \tau^{\Delta}$ is positive and non increasing on $\left[t_{0}, \infty\right)$ for some $t_{0} \in T$ and let $\tau(T)=T$. Then there exist an unbounded solution $\psi \in C_{r d}^{1}\left(\left[t_{0}, \infty\right)\right.$ of $(6)$ whit a positive and non increasing delta-derivative on $\left[\tau\left(t_{0}\right), \infty\right)$.

The question of the existence of a positive solution $\phi$ of (7) having certain additional properties can be dealt with similarly. In particular, if $a$ and $b$ are nonzero constant functions, then (7) becomes

$$
\forall t \in T, \quad|b| \phi(\tau(t)) \unlhd a \mid \phi(t)
$$

and $\phi(t)=\left|\frac{b}{a}\right|^{\psi(t)}$, where $\psi$ is a solution of (6), and this defines the positive function satisfying (8).

Consider the following equation:

$$
\forall t \in T, \quad x^{\Delta}(t)=a(t) x(t)+b(t) x(\tau(t)),
$$

J. Cermak and M. Urbanek have shown the following theorems and corollaries about asymptotic behavior of solutions of (9) in [5]:

Theorem 2.22 (see [5]). Consider Equation (9), where $a, b, \tau \in C_{r d}(T), a(t)>0, b(t) \neq 0$ for all $t \in T$ and let $\tau: T \rightarrow T$ be increasing on $T$ such that $\tau(t)<t$ for all $t \in T$ and $\lim _{t \rightarrow \infty} \tau(t)=\infty$. Further assume that

$$
\forall t_{0} \in T, \quad \int_{t_{0}}^{\infty}|b(t)| e_{a}(\tau(t), \sigma(t)) \Delta t<\infty
$$

then for any solution $x$ of (9) there exists a constant $L \in R$ such that 


$$
\lim _{t \rightarrow \infty} x(t) e_{a}\left(t_{0}, t\right)=L
$$

Lemma 2.23 (see [5]). Consider the equation

$$
t \in T, \quad x^{\Delta}(t)=a x(t)+b x(\tau(t))
$$

where $a>0, b \neq 0$ are real scalars and $\tau$ is a delta-differentiable on $T$ such that $\tau(t)<t$ for all $t \in T$, $\tau(T)=T$ and $0<\tau^{\Delta} \leq \lambda<1$ on $T$. Let $x$ be a solution of (12) satisfying (11) with $L=0$, i.e.,

$$
x(t)=O\left(e_{a}\left(t, t_{0}\right)\right), \quad \text { as } t \rightarrow \infty
$$

and let $\phi$ be a positive solution of (8), then

$$
x(t)=O(\phi(t))
$$

Theorem 2.24 (see [5]). Consider equation (12), where $a>0, b \neq 0$ are real scalars and $\tau$ is a deltadifferentiable function on $T$ such that $\tau(t)<t$ for all $t \in T, \tau(T)=T$ and $0<\tau^{\Delta} \leq \lambda<1$ on $T$. Then any solution $x$ of (12) satisfies (11). Moreover, if $x_{1}, x_{2}$ are solutions of (12) satisfying (11) with the same limit L. Then

$$
x_{1}(t)-x_{2}(t)=O(\phi(t)) \quad \text { as } t \rightarrow \infty,
$$

where $\phi$ is a positive solution of (8).

Now, we consider the equation (9) with a negative coefficient $a$.

Proposition 2.25 (see [5]). Let $a \in R^{+}, a(t) \leq \alpha<0$ for all $t \in T$ and let $\tau$ be a delta-differentiable function on $T$ such that $\tau(t)<t$ for all $t \in T, \tau(T)=T$ and $0<\tau^{\Delta} \leq \lambda<1$ on $T$. Then

$$
\mu(t) \equiv 0,
$$

on $T$.

Using proposition (2.25) we can derive the following consequence:

Corollary 2.26 (see [5]). Consider Dynamic equation (9), where $a \in R^{+}, b \in C_{r d}(T), \tau \in C_{r d}^{1}(T)$, a is non increasing on $T, a(t) \leq \alpha<0,0 \neq|b(t)| \leq \beta, \tau(t)<t$ for all $t \in T, \tau(T)=T, \tau^{\Delta}$ is positive and non increasing on $T$ and $\tau^{\Delta}\left(t_{0}\right)<1$. If $\psi$ is a solution of (6) with the properties guaranteed by Proposition (2.21)), then

$$
x(t)=O\left(\left(\frac{\beta}{-\alpha}\right)^{\psi(t)}\right) \quad \text { as } t \rightarrow \infty
$$

for any solution $x$ of (9). 


\section{Stability Results}

Theorem 3.1 Assuming that the assumptions of corollary (2.26) holds and $\beta<-\alpha$, then the solution $x(t)$ of (9) is asymptotically stable.

proof. According to definition (2.20), we must show that if $x(t)$ is a solution of (9), then

$$
\lim _{t \rightarrow \infty} x(t)=0
$$

From Proposition (2.21) it becomes clear $\psi$ that is a solution of (6) is an increasing and unbounded function. Also according to Corollary (2.26) we know that if $\beta<-\alpha$ then

$$
\phi(t)=\left(\frac{\beta}{-\alpha}\right)^{\psi(t)} \rightarrow 0 \quad \text { as } t \rightarrow \infty
$$

Then from Lemma (2.23) we see that

$$
x(t)=O(\phi(t))
$$

Thus we have

$$
\lim _{t \rightarrow \infty} x(t)=0
$$

Example 3.2 Let $T=R$ and consider the equation

$$
x^{\prime}(t)=a(t) x(t)+b(t) x(\tau(t)),
$$

that $\tau$ is a differential function on $I=\left[t_{0}, \infty\right), t_{0} \geq 1, a(t)=\frac{-t}{t+1}$ and $b(t)=\frac{t^{2}}{2 t^{2}+1}$, and let $\tau(t)<t$ for all $t \in I, \lim _{t \rightarrow \infty} \tau(t)=\infty$ and $0<\tau^{\prime}(t) \leq \lambda<1$ on $I$.

It can be shown clearly that $a(t)<0, a, b \in C(I), b(t) \neq 0$. Also we have

$$
a(t)<-1=\alpha, \quad|b(t)|<\frac{1}{2}=\beta .
$$

and $\beta<-\alpha$. Thus according to (2.26), $\phi(t) \rightarrow 0$, as $t \rightarrow \infty$.

$$
\Rightarrow \quad x(t)=O(\phi(t)) \rightarrow 0 \quad \text { as } t \rightarrow \infty
$$

that is, from Theorem (3.1), $x(t)$ is asymptotically stable.

\section{Acknowledgements}

The authors gratefully acknowledge the comments by an anonymous referee on the draft of the article. 


\section{References}

[1] M. Adivar, Y. N. Raffoul, "Stability and periodicity in dynamic delay equations", Comput. and Math. with Appl., Vol. 58 (2009), pp.264-272.

[2] M. Bohner, A. Peterson, "Dynamic Equations on Time Scales: An Introduction with Applications", Birkhauser, Boston, (2001).

[3] M. Bohner, G. Sh. Guseinov, "Improper integrals on time scales", m Dynam. Systems Appl. , Vol. 12 (2003), (1-2), pp.45-66.

[4] J. Cermak, and M. Urbanek, "On the asymptotics of solutions of delay dynamic equations on time scales", Math. and Comput. Model. Vol. 46 (2007), pp. 445-458.

[5] L. Erbe, A. Peterson, P. Rehak, "Comparison theorems for linear dynamic equation on time scales”, J. Math. Anal. Appl. Vol.275 (2002), pp. 418-438.

[6] H. Györi, G. Ladas, "Oscillation Theory of Delay Differential Equations", Oxford Science Publications, Oxford, (1991).

[7] S. Hilger, "Ein Mathkettenkalkul mit Anwendung auf Zentrumsmannigfaltigkeiten”, Ph.D thesis, Universitat Wurzburg, (1988).

[8] M. N. Kipins, I. S. Levitskaya, "Stability of Delay Difference and Differential Equations" Similarities ans Distinctions, (2007).

[9] M. Bohner, A. Peterson, "Advances in Dynamic Equations on Time Scales", Birkhauser, Boston, Mass, USA, (2003).

[10] F. Ghomanjani, F. Ghassabzade, "A Meshless Method for Solving Delay Differential Equation Using Radial Basis Funtions with Error Analysis", Journal of Mathematics and Computer Science, Vol.11, pp. 105-110, (2014). 